\title{
The energy conservation optimization design of the cutting edges of the twist drill based on Dijkstra's algorithm
}

\author{
Liangshan Xiong ${ }^{1}\left(\mathbb{D} \cdot \mathrm{Bin}^{\mathrm{Li}^{1}}\right.$
}

Received: 6 November 2014 / Accepted: 17 April 2015 /Published online: 27 June 2015

(C) The Author(s) 2015. This article is published with open access at Springerlink.com

\begin{abstract}
The energy conservation optimization design about geometrical shape and dimensions of the cutting edges (lips) of the twist drill is meant to determine the main cutting edge curve in the rake face that minimizes the drilling power. Dijkstra's algorithm is introduced and applied to serve the above purpose. The rake surface of the twist drill is discretized into a set of nodes and arcs between the adjacent nodes, which together compose a digraph. Cutting angles of elementary cutting tools on the lips of a twist drill are determined by the method of displaying planar image; those angles, together with undeformed chip thickness and width, are substituted into the empirical model of cutting force for elementary cutting tool to obtain the weights of arcs in the digraph, that is, the drilling power. Then, a main cutting edge curve in the rake face that minimizes the drilling power is generated using Dijkstra's algorithm. Meanwhile, an improvement in Dijkstra's algorithm procedures is put forward to reduce time and space complexities of the process and improve smoothness and machinability of the cutting edge curve. A computer program is developed with Matlab 2011b to determine the main cutting edge curve. The calculation results with $0.50 \%$ carbon steel show that the new curved cutting edge reduces the drilling power by 7 8\%, compared to a conventional straight cutting edge.
\end{abstract}

Keywords Twist drill $\cdot$ Drilling power $\cdot$ Energy conservation . Cutting lip $\cdot$ Shortest path $\cdot$ Dijkstra's algorithm

Liangshan Xiong

liangsx@hust.edu.cn

1 School of Mechanical Science \& Engineering, Huazhong University of Science and Technology, Wuhan, Hubei 430074, People's Republic of China

\section{Introduction}

Conventional twist drills are widely used in manufacturing, of which the flake is usually grinded into a conical surface and the main cutting edge (lip) a straight line. An enterprise in China may sell 1000,000,000 conventional high-speed steel (HSS) twist drills annually. However, their drilling performance is not satisfactory. Related problems are many and various, among which is the undesired distribution of the cutting angles of the main cutting edge due to the limitation of the grinding structure and the grinding method, resulting in poor drilling performance and a higher drilling power [1]. For a long time, people have been making huge effort to reduce the energy consumption in drilling by improving the grinding structure of the drill point [1-4]. Among the numerous kinds of improved drills, the most outstanding is the multifacet drill (MFD), which is called the Ni Zhifu drill in China. It is reported that the MFD reduces the energy consumption by $20 \%$ compared to the standard twist drill when drilling plain carbon steel $[1,2]$.

Unfortunately, the MFD is difficult to be marketed and has a limited range of application because of its especially complicated grinding method. Under the instruction of the Plannar Display Theory $[5,6]$, which aims at mapping of images on a projection plane, Xiong and Shi et al. [7, 8] put forward a curved cutting edge design that controls the distribution of rake angles of the main cutting edge by changing the orientation of the edge, and thus reduces its energy consumption in drilling. This design discards the requirement of straight-line cutting edge of the standard grinding process of the conventional twist drill (without changing its manufacturing process and the original structure). A curve-edged drill that maximizes the value of the rake angle at each point along the tool cutting edge (hereafter referred to as the "drill with large rake angles") is developed, the flank face of which is grinded as a simple 
ruled surface $[9,10]$. In this way, grinding problems that block the marketing of the optimized drill are well solved and a new path for energy conservation optimization of the drill tip grinding structure is opened. The drilling torque is reduced by about $12-20 \%$ compared to conventional drills, with remarkable energy-saving effect. Xiong [11], Xiong and Fang et al. [12] further introduce the specific method to optimize the main cutting edge curve and reduce the energy consumption in cutting based on certain given cutting angle distributions of the main cutting edge, such as rake angle distribution, inclination angle distribution, tool cutting edge angle distribution, etc. As is well-known, the cutting power of cutting tools is not only affected by the cutting angle distribution but also by undeformed chip thickness, undeformed chip width, and cutting parameters. Change in the orientation of the main cutting edge will also cause change in cutting angles and in undeformed chip thickness and width. Nevertheless, changes in the three elements may not be in the same direction, and thus may result in heterogeneous change in cutting energy consumption. Therefore, it is almost impossible for the optimized main cutting edge curve based on any randomly given cutting angle distribution to be the most energy-saving curve. The problem is that the given cutting angle distribution is very unlikely to be the optimal distribution. Then what kind of cutting angle distribution is the optimal one? In other words, what kind of main cutting edge curve is the most energysaving?

The question is essentially how to determine the main cutting edge curve in the helicoid rake face of the twist drill that minimizes the drilling power, which is a typical functional optimization problem. However, "functional analysis" [13] has not provided a general method to determine the functional extremums of such a curve on a hook surface.

From a mathematical perspective, the abovementioned problem is of the same nature with the determination with a traffic navigation software of the shortest path between any two points in a given traffic network. Based on this insight, this essay makes an attempt to apply Dijkstra's algorithm [14-17] which settles the shortest path problem of the graph theory to the energy conservation optimization of the main cutting edge curve of the twist drill. A specific method used in determining the main cutting edge curve in the helicoid rake face of the twist drill that minimizes the drilling power is introduced. The idea is as follows: The rake surface of the twist drill is discretized into a set of nodes and arcs between the adjacent nodes, which together compose a digraph. The arc between any adjacent nodes (hereafter referred to as "ECT" standing for "elementary cutting tool") is a possible part of the main cutting edge curve with the minimum drilling power (hereafter referred to as "MCECMDP"); relational models of ECT cutting forces with cutting angles and cutting parameters are established through cutting experiments; the cutting angles and the cutting parameters of the ECTs that constitute the main cutting edge curve of the twist drill are figured out; the drilling forces are calculated through the above models, and then the drilling power can be obtained; the ECT drilling power being the weight of arc in the rake surface digraph, the shortest path between the source and the destination composed of adjacent arcs, the sum of whose weights is the minimal, is located through Dijkstra's algorithm. This path is exactly the MCECMDP. Programmed calculation shows that the MCECMDP is not the curve with the largest rake angles; when machining a $0.50 \%$ carbon steel workpiece with a drill with a diameter of $\phi 25 \mathrm{~mm}$, the drilling power of a MCECMDP is reduced by $7.45 \%$ compared to that of the straight main cutting edge of a standard twist drill.

\section{Introduction to the shortest path problem and Dijkstra's algorithm}

\subsection{The shortest path problem}

The shortest path problem is a classical topic in graph theory researches. The essence of the issue is to find out the shortestdistance path between two nodes vertex on a digraph $[13,14]$ $G=(V, E)$ ( $V$ is the set of all the vertices (nodes), and $E$ is the set of weighted edges that connect the nodes), that is, to find out the path composed of adjacent edges, of which the sum of weights is minimized.

When a source node $v_{\mathrm{s}}$ in $V$ is given and the arc weights in $G$ are all nonnegative real numbers, the shortest path problem that aims to find the shortest distances from $v_{\mathrm{s}}$ to all other nodes is referred to as the single-source shortest path problem.

\subsection{Dijkstra's algorithm}

Dijkstra's algorithm is a classical algorithm to solve the single-source shortest path problem.

The solution pattern of Dijkstra's algorithm is as follows: to divide $\mathrm{V}$ into two sets $\mathrm{S}$ and $\mathrm{T}$. $\mathrm{S}$ is the set of all the ending nodes of the determined shortest paths to the source node $v_{\mathrm{s}}$; at the initial stage, $\mathrm{S}$ only contains $v_{\mathrm{s}}$. T is the set of nodes of the undetermined shortest paths to the source node $v_{\mathrm{s}}$. Nodes in T will be moved to $\mathrm{S}$ in an ascending order of the length of the shortest paths to the source node $v_{\mathrm{s}}$, until there is no node left in set $\mathrm{T}$. The path composed by all the edges that connect the source node $v_{\mathrm{S}}$ to any node $v_{\mathrm{t}}$ in order is the shortest path from $v_{\mathrm{s}}$ to $v_{\mathrm{t}}$. The sum of the corresponding weight values is the length of the shortest path.

Many practical problems can be abstracted and then transformed into the shortest path problem, such as location of the optimal route in the road traffic network, the optimal transmission of information flow among routers in the computer network, etc. Therefore, Dijkstra's algorithm has been successfully applied in such fields [16] as operational research, 
computer science, geographic information science and transportation, etc.

\section{Adjacency matrix of the rake face network}

To determine the main cutting edge curve of the twist drill with the minimal drilling power is to find a curve on the helicoid rake face that starts from any point on the tool minor cutting edge, for instance the outer corner $\mathrm{C}$, to a point on the cylindrical spiral passing the chisel edge corner $\mathrm{B}$ that ensures the consumed drilling power reaches its minimum when this curve serves as the main cutting edge. Obviously, this is a typical single-source shortest path problem. In order to solve this problem with Dijkstra's algorithm, the rake surface of the twist drill should be discretized into a set of nodes and edges between adjacent nodes, which together compose a digraph (network). Also, the edge weights should be evaluated to establish an adjacency matrix of the network.

\subsection{Equation set of the rake face of the twist drill}

The rake face of the twist drill corresponding to the main cutting edge is a helicoid formed through the helical motion of the straight generating line round the axis of the drill. Assuming the drill diameter is $D$, in the drill structure coordinate system $O x y z$ shown in Fig. 1, the equations of the rake face corresponding to the straight main cutting edge $\mathrm{BC}$ are

$$
\left\{\begin{array}{l}
x=\sqrt{r^{2}-r_{0}^{2}} \cos \varepsilon+r_{0} \sin \varepsilon \\
y=\sqrt{r^{2}-r_{0}^{2}} \sin \varepsilon-r_{0} \cos \varepsilon \\
z=\sqrt{r^{2}-r_{0}^{2}} / \tan \varphi_{0}+T \varepsilon /(2 \pi)
\end{array}\right.
$$

where $2 \varphi_{0}$ is the drill point angle, $2 \varphi_{0}=118^{\circ} ; T$ is the pitch of the drill flute helicoid, $T=2 \pi \mathrm{R} / \tan \beta$, in which $R=0.5 D$ is the drill radius, $\beta$ is the helical angle in the outer corner $\mathrm{C}, \beta=30^{\circ}$; $r$ is the radius of any point on the rake face; and $\varepsilon$ is the turning angle of the point.

The coordinates of points $\mathrm{C}$ and $\mathrm{B}$ are, respectively, $\left(\sqrt{R^{2}-r_{0}^{2}},-r_{0}, \sqrt{R^{2}-r_{0}^{2}} / \tan \varphi_{0}\right)$, and $\left(r_{0} / \tan \psi,-r_{0}, 0\right)$.

\subsection{Discretization of the rake face}

As is shown in Fig. 2, the rake face is discretized into a directed network through the following procedures:

1. In the $x O y$ plane, the rake face's projection $\mathrm{ABCD}(\mathrm{BC}$ is the projection of the main cutting edge of the standard twist drill in the $x O y$ plane; $\mathrm{AD}$ and $\mathrm{BC}$ are parallel and there, distance to $x$ axis is $r_{01}$ ) and is divided into $n-1$ arc segments by $n$ concentric circles, of which the radiuses are
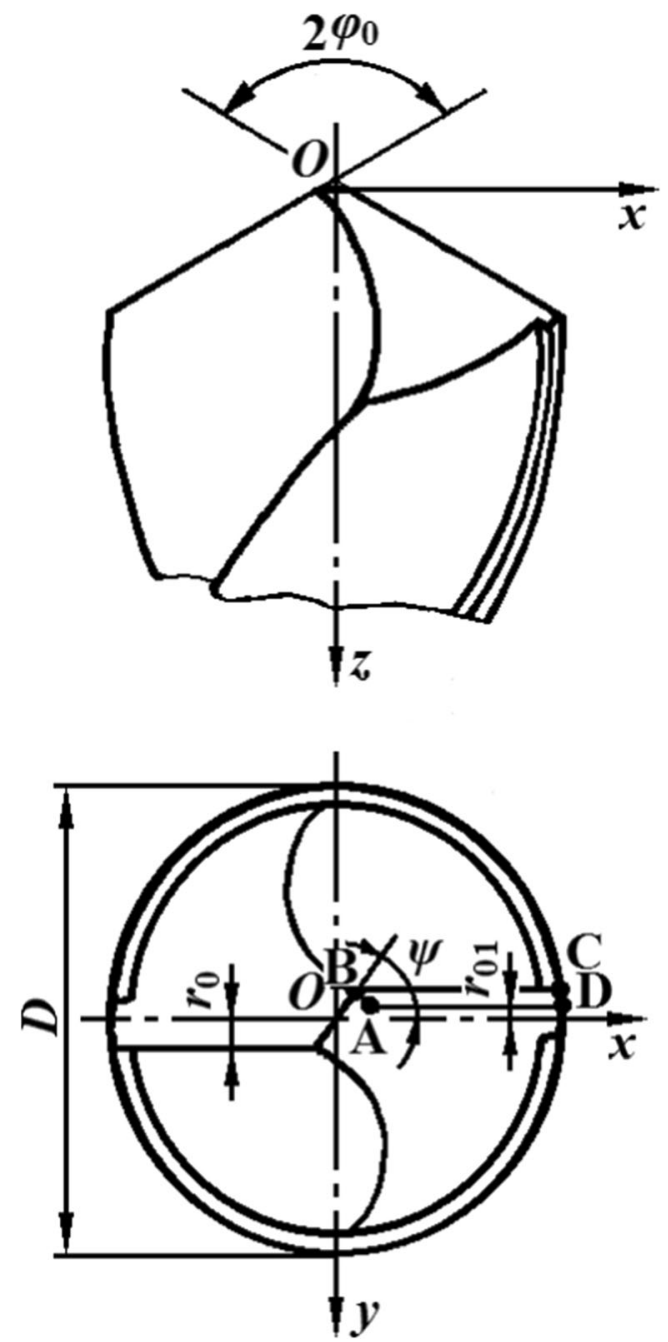

Fig. 1 The drill structure coordinate system

distributed with arithmetic progression and the center is $O$, the origin of coordinates. The radius of the $i$ th concentric circle is

$$
r_{i}=R-(i-1)\left(R-r_{0} / \sin \psi\right) /(n-1), \quad i=1,2,3 \ldots n
$$

where $\psi$ is the chisel edge angle of the conventional twist drill. Correspondingly, the rake face is divided into $n-1$ strips of helicoid by $n$ co-axial cylindrical surfaces.

2. Each arc between $\mathrm{AD}$ and $\mathrm{BC}$ is divided into $m-1$ equal segments (so the grids in the rake face are different in

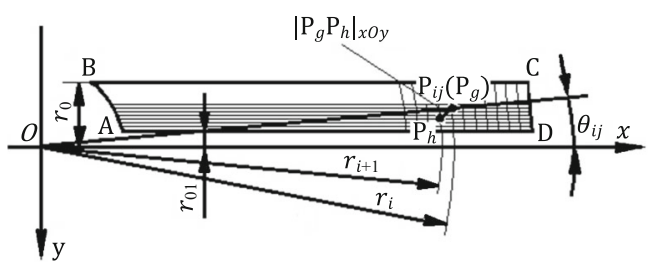

Fig. 2 Discretization of the rake face 
size). Assuming $j$ is the equipartition point number, for the points on $\mathrm{AD}, j=1$, and for the points on $\mathrm{BC}, j=m$. Therefore, $n m$ (i.e. $n \times m$ ) nodes are obtained. The $j$ th node on the $i$ th arc is marked as $\mathrm{P}_{i j}$, and the angle between $O \mathrm{P}_{i j}$ and $x$ axis is

$$
\begin{aligned}
\theta_{i j}= & (j-1)\left(\tan ^{-1}\left(\frac{r_{0}}{r_{i}}\right)-\tan ^{-1}\left(\frac{r_{01}}{r_{i}}\right)\right) /(m-1) \\
& +\tan ^{-1}\left(\frac{r_{01}}{r_{i}}\right), j=1,2,3 \ldots m
\end{aligned}
$$

Assuming the coordinates of $\mathrm{P}_{i j}$ are $\left(x_{i j}, y_{i j}, z_{i j}\right)$, since $\mathrm{P}_{i j}$ is on the rake face, the coordinates can be obtained based on the related geometrical relations and the equation set (1):

$x_{i j}=r_{i} \cos \theta_{i j}, y_{i j}=r_{i} \sin \theta_{i j}, z_{i j}=t_{i} / \tan \varphi_{0}+T \varepsilon /(2 \pi)$

where $t_{i}=\sqrt{r_{i}^{2}-r_{0}^{2}}$ and the parameter $\varepsilon$ can be determined through the equation set (1) based on the values of the coordinates $\left(x_{i j}, y_{i j}\right)$ :

$$
\begin{aligned}
{\left[\begin{array}{c}
\cos \varepsilon \\
\sin \varepsilon
\end{array}\right] } & =\left[\begin{array}{cc}
t_{i} & r_{0} \\
-r_{0} & t_{i}
\end{array}\right]^{-1}\left[\begin{array}{c}
x_{i j} \\
-y_{i j}
\end{array}\right] \\
& =\left[\begin{array}{cc}
t_{i} & r_{0} \\
-r_{0} & t_{i}
\end{array}\right]^{-1}\left[\begin{array}{c}
r_{i} \cos \theta_{i j} \\
-r_{i} \sin \theta_{i j}
\end{array}\right]
\end{aligned}
$$

3. For programming convenience, all the nodes are renumbered from 1 to $n m$. Assume node $\mathrm{P}_{i j}$ is renumbered $g$, and node $\mathrm{P}_{(i+1) k}$ is renumbered $h$, then

$g=(i-1) m+j, h=i m+k$.

Hereafter, $\mathrm{P}_{g}$ and $\mathrm{P}_{h}$ are used to represent the same nodes $\mathrm{P}_{i j}$ and $\mathrm{P}_{(i+1) k}$ on the condition that no misunderstanding would be caused.

4. Connect node $\mathrm{P}_{g}$ and node $\mathrm{P}_{h}$. If the projection length $l_{i j k}$ of the line segment $\mathrm{P}_{g} \mathrm{P}_{h}$ in the $x O y$ plane satisfies

$$
l_{i j k}=\left|\mathrm{P}_{g} \mathrm{P}_{h}\right|_{x O y}=\sqrt{\left(x_{g}-x_{h}\right)^{2}+\left(y_{g}-y_{h}\right)^{2}} \leq \Delta r_{i}
$$

where

$$
\Delta r_{i}=\sqrt{r_{i}^{2}-r_{i+1}^{2}}, i=1,2,3 \ldots \ldots n-1
$$

In other words, if there is one, but only one intersection between the $i+1$ th arc and the line segment $\mathrm{P}_{g} \mathrm{P}_{h}$ obtained by connecting node $\mathrm{P}_{i j}$ and node $\mathrm{P}_{(i+1) k}$, then line $\mathrm{P}_{g} \mathrm{P}_{h}$ is one edge of the directed network (digraph) of the rake face.

\subsection{Adjacency matrix}

The adjacency matrix $\boldsymbol{A}$ of the directed network of the rake face is a $n m \times n m$ square matrix. Its element $w_{g h}(g=1,2,3 \ldots$ $n m ; h=1,2,3 \ldots n m)$ is the weight value of the edge that connects the $g$ th and $h$ th nodes.

Any edge in the directed network of the rake face may be a segment of the MCECMDP that is to be determined. The drill part that corresponds to the edge $\mathrm{P}_{g} \mathrm{P}_{h}$ viewed as an ECT that can operate cutting independently, its cutting edge is the edge $\mathrm{P}_{g} \mathrm{P}_{h}$, and its rake face is the tangent plane to the rake face at the midpoint of this edge. If the edge is short enough, it can be substituted by the straight line that connects its ends. Since it is the main cutting edge with the minimal drilling power that is to be determined, the cutting power (indicated as $\Delta P_{g h}$ ) of the ECT is defined as the weight of the edge $\mathrm{P}_{g} \mathrm{P}_{h}$.

$w_{g h}=\Delta P_{g h}, \quad\left|\mathrm{P}_{g} \mathrm{P}_{h}\right|_{x O y} \leq \Delta r_{i}$

If the length of the edge that connects node $\mathrm{P}_{g}$ and node $\mathrm{P}_{h}$ does not satisfy the Eq. (7), it means there exists no such edge that can connect these two nodes. In this case, the corresponding cutting power $\Delta P_{g h}$ of the ECT would be infinite. Therefore,

$w_{g h}=\left\{\begin{array}{l}\Delta P_{g h}, \quad\left|\mathrm{P}_{g} \mathrm{P}_{h}\right|_{x O y} \leq \Delta r_{i} \\ \infty, \quad\left|\mathrm{P}_{g} \mathrm{P}_{h}\right|_{x O y}>\Delta r_{i}\end{array}\right.$

By calculating the drilling powers of all the ECTs, that is, the weights of all edges, the adjacency matrix $\boldsymbol{A}$ of the rake face discrete network (digraph) can be obtained.

\section{Calculation of edge weight}

The terminologies and definitions employed in this section follow those recommended by ISO $3002[18,19]$.

In order to determine the cutting power of the ECT, its cutting angles and its undeformed chip thickness and width should be calculated first.

\subsection{ECT cutting angles}

ECT cutting angles are defined as the cutting angles of the main cutting edge of the twist drill at the midpoint of the ECT cutting edge, including the working rake angle $\gamma_{0 e}$, the working normal rake angle $\gamma_{\text {ne }}$, the working tool inclination angle $\lambda_{\text {se }}$, and the working cutting edge angle $\kappa_{\text {re }}$. The method of displaying planar image $[5,6]$ is applied to determine the ECT cutting angles.

\subsubsection{Formation of image points and image lines on the projection plane}

Assume the coordinates of the end points of the edge $\mathrm{P}_{g} \mathrm{P}_{h}$ to be $\left(x_{g}, y_{g}, z_{g}\right)$ and $\left(x_{h}, y_{h}, z_{h}\right)$. The coordinates $\left(x_{g h m}, y_{g h m}\right.$, 
$\left.z_{g h m}\right)$ and the radius of its midpoint $\mathrm{P}_{i m}$ are, respectively, as follows:

$$
\left\{\begin{array}{c}
x_{g h m}=\frac{x_{g}+x_{h}}{2}, y_{g h m}=\frac{y_{g}+y_{h}}{2}, z_{g h m}=\frac{z_{g}+z_{h}}{2} \\
r_{g h m}=\frac{r_{g}+r_{h}}{2}=\frac{r_{i}+r_{i+1}}{2}
\end{array}\right.
$$

Assume the rotation speed of the twist drill to be $N / \mathrm{r} \bullet \mathrm{s}^{-1}$ and its feed to be $f / \mathrm{m}^{\circ} \mathrm{r}^{-1}$. The image points and image lines can be created in the projection plane according to the method of displaying planar image $[5,6]$, as shown in Fig. 3. $d$ in the figure is the image line of the tangent line (which is substituted by line $\mathrm{P}_{g} \mathrm{P}_{h}$ ) of the ECT cutting edge at its midpoint; $\boldsymbol{Q}, \boldsymbol{P}_{\mathbf{e}}, \boldsymbol{T}_{\mathbf{e}}, \boldsymbol{R}_{\mathbf{e}}, \boldsymbol{N}, \boldsymbol{F}$ are, respectively, the image points of the rake face, the working reference plane, the working cutting edge plane, the working orthogonal plane, the cutting edge normal plane, and the assumed working plane at this midpoint. Based on the definitions of these planes and their relations and the Eq. set (1) for the rake face, the homogeneous coordinates $[5,6,8]$ of these images can be obtained.

$\boldsymbol{d}=\left[\begin{array}{lll}x_{h}-x_{g} & y_{h}-y_{g} & z_{h}-z_{g}\end{array}\right]^{\mathrm{T}}=\boldsymbol{N}$,

$\boldsymbol{Q}=\left[\begin{array}{c}-\frac{d y}{d x} \\ \left.\right|_{d z=0} \\ -\left.\frac{d y}{d z}\right|_{d x=0}\end{array}\right]$

$$
=\left[\begin{array}{c}
\frac{T \sin \varepsilon \tan \varphi_{0}-2 \pi r_{0} \sin \varepsilon-2 \pi \sqrt{r_{i m}^{2}-r_{0}^{2}} \cos \varepsilon}{-T \cos \varepsilon \tan \varphi_{0}+2 \pi r_{0} \cos \varepsilon-2 \pi \sqrt{r_{i m}^{2}-r_{0}^{2}} \sin \varepsilon} \\
1 \\
\frac{2 \pi\left(r_{0} \cos \varepsilon-\sqrt{r_{i m}^{2}-r_{0}^{2}} \sin \varepsilon\right) \tan \varepsilon-2 \pi r_{0} \sin \varepsilon-2 \pi \sqrt{r_{i m}^{2}-r_{0}^{2}} \cos \varepsilon}{T+2 \pi\left(\sqrt{r_{i m}^{2}-r_{0}^{2}} \sin \varepsilon-r_{0} \cos \varepsilon\right) /\left(\cos \varepsilon \tan \varphi_{0}\right)}
\end{array}\right]
$$

$\boldsymbol{P}_{\mathbf{e}}=\boldsymbol{v}_{\mathbf{e}}=\left[\begin{array}{lll}-\pi\left(z_{g}+z_{h}\right) & -f & \pi\left(x_{g}+x_{h}\right)\end{array}\right]^{\mathrm{T}}$,

$T_{\mathrm{e}}=d \times v_{\mathrm{e}}$,

$\boldsymbol{R}_{\mathrm{e}}=\boldsymbol{P}_{\mathrm{e}} \times \boldsymbol{T}_{\mathrm{e}}$,

and

$\boldsymbol{F}=\left[\begin{array}{lll}-\left(x_{g}+x_{h}\right) & 0 & \left(z_{g}+z_{h}\right)\end{array}\right]^{\mathrm{T}} / 2$,

where $v_{\mathbf{e}}$ is the image line of the cutting velocity $v_{\mathbf{e}}$ at the midpoint.

\subsubsection{Calculation of the cutting angles}

According to the method of displaying planar image [5, 6], the Euclidean angle $\theta_{\mathrm{ACB}}$ between the planes $\mathrm{A}$ and $\mathrm{B}$ which is measured in the plane $\mathrm{C}$ is equal to the non-
Euclidean angle $\theta_{\boldsymbol{A} \boldsymbol{C} \boldsymbol{B}}$ formed by the image points $\mathrm{A}, \mathrm{B}$, and $\mathrm{C}$. Based on the homogeneous coordinates, their value can be obtained $[5,6]$ :

$\theta_{A C B}=\theta_{\boldsymbol{A} \boldsymbol{C} \boldsymbol{B}}=\tan ^{-1}\left(\sqrt{\boldsymbol{C}^{\mathrm{T}} \boldsymbol{C}}\left|\begin{array}{lll}A_{1} & A_{2} & A_{3} \\ B_{1} & B_{2} & B_{3} \\ C_{1} & C_{2} & C_{3}\end{array}\right| /\left|\begin{array}{ll}\boldsymbol{C}^{\mathrm{T}} \boldsymbol{C} & \boldsymbol{C}^{\mathrm{T}} \boldsymbol{A} \\ \boldsymbol{B}^{\mathrm{T}} \boldsymbol{C} & \boldsymbol{B}^{\mathrm{T}} \boldsymbol{A}\end{array}\right|\right)$.

Substituting the homogeneous coordinates obtained from Eqs. (12) (17) in Eq. (18) yields the values of all the cutting angles, that is, the working rake angle $\gamma_{0 \mathrm{e}}$, the working normal rake angle $\gamma_{\mathrm{n}}$, the working tool inclination angle $\lambda_{\mathrm{s} e}$, and the working cutting edge angle $\kappa_{\text {re }}$ Fig. 3:

$\left\{\begin{array}{l}\gamma_{0 \mathrm{e}}=\theta_{\boldsymbol{Q} \boldsymbol{R}_{\mathrm{e}} \boldsymbol{P}_{\mathrm{e}}} \\ \gamma_{\mathrm{n} e}=\theta_{\boldsymbol{Q N} \boldsymbol{P}_{\mathrm{e}}} \\ \lambda_{\mathrm{s} e}=\theta_{\boldsymbol{Q} \boldsymbol{T}_{\mathrm{e}} \boldsymbol{P}_{\mathrm{e}}} \\ \kappa_{\mathrm{r} e}=\theta_{\boldsymbol{F} \boldsymbol{P}_{\mathrm{e}} \boldsymbol{T}_{\mathrm{e}}}\end{array}\right.$

\subsection{Undeformed chip thickness and cut width}

The undeformed chip thickness $a_{\mathrm{cgh}}$ and the cut width $a_{\mathrm{wgh}}$ of the ECT are, respectively, $[1,8]$

$a_{\mathrm{c} g h}=f \sin \kappa_{\mathrm{regh}} v_{g h} /\left(2 v_{\mathrm{egh}}\right)$,

and $[1,8]$

$a_{\mathrm{w} g h}=d l_{g h} \cos \lambda_{\mathrm{segh}}$,

where $\kappa_{\text {regh }}$ and $\lambda_{\text {segh }}$ are, respectively, the working cutting edge angle and the working tool inclination angle of the

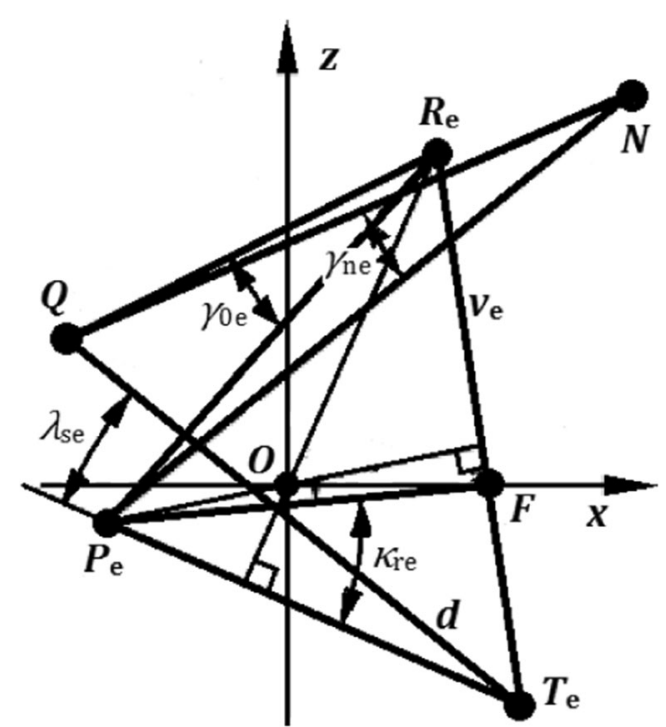

Fig. 3 Angular and distance relationships among relevant image points and image lines on the projection plane [12] 
ECT; $v_{g h} / v_{\mathrm{egh}}$ is the ratio of the rotational speed $v_{g h}$ to cutting velocity $v_{\text {egh }}$, and

$v_{g h} / v_{\mathrm{e} g h}=\sqrt{\left(x_{g h m}\right)^{2}+\left(y_{g h m}\right)^{2}} / \sqrt{\left(x_{g h m}\right)^{2}+\left(\frac{f}{2 \pi}\right)^{2}+\left(y_{g h m}\right)^{2}}$,

$d l_{g h}$ is the length of the ECT cutting edge, and

$d l_{g h}=\sqrt{\left(x_{h}-x_{g}\right)^{2}+\left(y_{h}-y_{g}\right)^{2}+\left(z_{h}-z_{g}\right)^{2}}$.

\subsection{Empirical models of ECT cutting forces}

The ECT cutting forces are calculated with the empirical relational models of the cutting forces with the cutting angles, the undeformed chip thickness, and the cut width that are obtained through cutting experiments.

\subsubsection{Turning experiments on the end face of sleeve parts}

A set of turning experiments with HSS end-cut tools on $0.50 \%$ carbon steel were designed, which adopt the L25 $\left(5^{6}\right)$ orthogonal array based on the three-factor experimental results so as to establish the empirical models of the cutting forces. The experiments were conducted on a CM6140-type horizontal lathe. The objects to be machined are turned $0.50 \%$ carbon steel sleeve parts (with a hardness of HBS241, an outer diameter of $\phi 115 \mathrm{~mm}$, and an inner diameter of $\phi 110 \mathrm{~mm}$, Fig. 4). The cutting forces were measured by a Kistler threecomponent piezoelectric dynamometer with the computer data acquisition system. Altogether, 25 sets of HSS end-cut tools (with the cutting edge angle $\kappa_{\mathrm{r}}=90^{\circ}$ ) with different rake angles and inclination angles were grinded, and a second round of cutting experiments were made, in which the cutting tools were resharpened. Due to limited space, Table 1 shows the cutting forces measured in the first round only, where $\gamma_{0}$ is the rake angle; $\lambda_{\mathrm{s}}$ is the inclination angle; $f$ is the feed per spindle

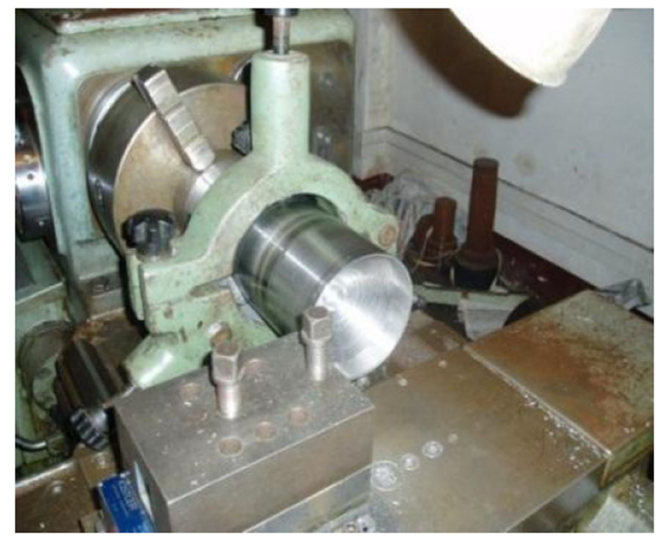

Fig. 4 Face cutting experiments on sleeve parts
Table 1 Results of face cutting experiments on sleeve parts

\begin{tabular}{|c|c|c|c|c|c|c|c|}
\hline No. & $\gamma_{0} /^{\circ}$ & $\lambda_{\mathrm{s}}{ }^{\circ}$ & $f / \mathrm{mm} \bullet \mathrm{r}^{-1}$ & $v_{\mathrm{e}} / \mathrm{m} \cdot \min ^{-1}$ & $F_{\mathrm{S}} / \mathrm{N}$ & $F_{\mathrm{o}} / \mathrm{N}$ & $F_{\mathrm{r}} / \mathrm{N}$ \\
\hline 1 & -30 & -30 & 0.04 & 3.5 & 163.2 & 31.8 & 209.5 \\
\hline 2 & -30 & -15 & 0.05 & 7.1 & 140.7 & 34.8 & 245.2 \\
\hline 3 & -30 & 0 & 0.065 & 14.1 & 371.8 & 14.0 & 352.5 \\
\hline 4 & -30 & 15 & 0.08 & 22.3 & 457.0 & -52.3 & 326.9 \\
\hline 5 & -30 & 30 & 0.1 & 28.3 & 568.0 & -80.4 & 381.5 \\
\hline 6 & -15 & -30 & 0.05 & 14.1 & 127.7 & 62.8 & 218.5 \\
\hline 7 & -15 & -15 & 0.065 & 22.3 & 393.4 & 57.5 & 349.4 \\
\hline 8 & -15 & 0 & 0.08 & 28.3 & 406.1 & 12.2 & 343.8 \\
\hline 9 & -15 & 15 & 0.1 & 3.5 & 255.2 & -29.5 & 390.2 \\
\hline 10 & -15 & 30 & 0.04 & 7.1 & 110.1 & -41.8 & 182.1 \\
\hline 11 & 0 & -30 & 0.065 & 28.3 & 262.4 & 79.2 & 296.7 \\
\hline 12 & 0 & -15 & 0.08 & 3.5 & 175.1 & 40.7 & 316.2 \\
\hline 13 & 0 & 0 & 0.1 & 7.1 & 138.7 & 17.4 & 291.4 \\
\hline 14 & 0 & 15 & 0.04 & 14.1 & 84.0 & -10.9 & 151.4 \\
\hline 15 & 0 & 30 & 0.05 & 22.3 & 126.6 & -50.7 & 196.6 \\
\hline 16 & 15 & -30 & 0.08 & 7.1 & 91.6 & 59.1 & 250.6 \\
\hline 17 & 15 & -15 & 0.1 & 14.1 & 125.6 & 41.4 & 273.3 \\
\hline 18 & 15 & 0 & 0.04 & 22.3 & 71.1 & 4.4 & 132.0 \\
\hline 19 & 15 & 15 & 0.05 & 28.3 & 87.3 & -16.8 & 161.9 \\
\hline 20 & 15 & 30 & 0.065 & 3.5 & 77.8 & -50.2 & 198.5 \\
\hline 21 & 30 & -30 & 0.1 & 22.3 & 84.2 & 84.1 & 271.6 \\
\hline 22 & 30 & -15 & 0.04 & 28.3 & 51.7 & 19.1 & 115.9 \\
\hline 23 & 30 & 0 & 0.05 & 3.5 & 39.2 & 5.0 & 130.1 \\
\hline 24 & 30 & 15 & 0.065 & 7.1 & 55.8 & -13.4 & 155.4 \\
\hline 25 & 30 & 30 & 0.08 & 14.1 & 103.1 & -81.5 & 232.7 \\
\hline
\end{tabular}

revolution; $v_{\mathrm{e}}$ is the working cutting speed, which is calculated based on the rotation speed and the workpiece pitch diameter (with the feed speed ignored); $F_{\mathrm{s}}$ is the axial thrust force; $F_{\mathrm{o}}$ is the radial thrust force, and $F_{\mathrm{r}}$ is the main cutting force.

\subsubsection{Empirical models of cutting forces}

The experimental data were imported to the statistical analysis software EView [20] so that the following empirical relational formulas of the main cutting force $F_{\mathrm{r}}$, the axial thrust force $F_{\mathrm{s}}$, and the radial thrust force $F_{\mathrm{o}}$ with the cutting angles, the undeformed chip thickness, and the cut width were fitted:

$$
\left\{\begin{array}{c}
F_{\mathrm{r}}=2.567 \times 10^{8} a_{\mathrm{w}} a_{\mathrm{c}}^{0.8223} \exp \left(-0.6204 \gamma_{\mathrm{n}}-0.4271 \lambda_{\mathrm{s}}^{2}\right) \mathrm{N} \\
F_{\mathrm{s}}=1.323 \times 10^{8} a_{\mathrm{w}} a_{\mathrm{c}}^{0.9455}\left(\cos \lambda_{\mathrm{s}}\right)^{1.351} v_{\mathrm{e}}^{0.2121} \exp \left(1.720\left(1-\sin \gamma_{\mathrm{n}}\right)\right) \mathrm{N} \\
F_{\mathrm{o}}=-5.000 \times 10^{3} a_{\mathrm{w}}\left(-3.461 \times 10^{5} a_{\mathrm{c}}+1.739 \times 10^{10} a_{\mathrm{c}}^{2}-2.509 \times 10^{14} a_{\mathrm{c}}^{3}\right. \\
\left.+1.208 \times 10^{18} a_{\mathrm{c}}^{4}-4.817 \gamma_{\mathrm{n}}^{2}+3.730 \gamma_{\mathrm{n}}^{3}-0.824 \lambda_{\mathrm{s}}\right) \mathrm{N}
\end{array}\right.
$$

where the unit of the cutting angles (including $\gamma_{\mathrm{n}}$ and $\lambda_{\mathrm{s}}$ ) is radian, and $\gamma_{\mathrm{n}}=\tan ^{-1}\left(\tan \gamma_{0} \cos \lambda_{\mathrm{s}}\right) ; a_{\mathrm{w}}$ is the cut width, and for the given experimental condition $a_{\mathrm{w}}=2.5 \times 10^{-3} / \cos \lambda_{\mathrm{s}}$ (in meters); $a_{\mathrm{c}}$ is the undeformed chip thickness in meters, and $a_{\mathrm{c}}=f$; $v_{\mathrm{e}}$ is in meters per second $\left(\mathrm{m} \bullet \mathrm{s}^{-1}\right)$. 
In end-face turning, the differences between the cutting angles $\left(\gamma_{\mathrm{n}} \lambda_{\mathrm{s}}\right.$ and $\left.\kappa_{\mathrm{r}}\right)$ and the working cutting angles $\left(\gamma_{\text {ne }}, \lambda_{\text {se }}\right.$ and $\left.\kappa_{\text {re }}\right)$ are negligible. Therefore, the latter are substituted for the former in calculation in the following sections.

\subsection{ECT cutting forces}

The above empirical formulas are established on the condition that the cutting edge angle $\kappa_{\mathrm{r}}$ is $90^{\circ}$. Since no consideration is given to the influence of the change in the angle on the cutting force while in practice the angle may change along the main cutting edge, Eq. (24) cannot be directly used to calculate the drilling forces of the ECTs which constitute the main cutting edge. Therefore, in reference to [21], it is assumed that

$\mathbf{R}=\mathbf{R}_{1} \mathbf{R}_{2} \mathbf{R}_{3}$, and $\mathbf{R}_{0}=\mathbf{R}_{4} \mathbf{R}_{2} \mathbf{R}_{3}$

where

$\left.\begin{array}{rl}\mathbf{R}_{1} & =\left(\begin{array}{lll}1 & 0 & 0 \\ 0 & 1 & 0 \\ 0 & 0 & 1\end{array}\right) ; \mathbf{R}_{2}=\left(\begin{array}{ccc}1 & 0 & 0 \\ 0 & \cos \lambda_{\mathrm{se}} & -\sin \lambda_{\mathrm{se}} \\ 0 & \sin \lambda_{\mathrm{se}} & \cos \lambda_{\mathrm{se}}\end{array}\right) \\ \mathbf{R}_{3} & =\left(\begin{array}{ccc}\cos \gamma_{\mathrm{ne}} & 0 & -\sin \gamma_{\mathrm{ne}} \\ 0 & 1 & 0 \\ \sin \gamma_{\mathrm{ne}} & 0 & \cos \gamma_{\mathrm{ne}}\end{array}\right) ; \mathbf{R}_{4}=\left(\begin{array}{ccc}\sin \kappa_{\mathrm{re}} & -\cos \kappa_{\mathrm{re}} & 0 \\ \cos \kappa_{\mathrm{re}} & \sin \kappa_{\mathrm{re}} & 0 \\ 0 & 0 & 1\end{array}\right)\end{array}\right\}$.

Then, plug into the following equation the cutting forces $\Delta F_{\mathrm{s}} 、 \Delta F_{\mathrm{o}} 、 \Delta F_{\mathrm{r}}$ predicted by Eq. (24) when the cutting edge angle is $90^{\circ}$, the normal rake angle is $\gamma_{\text {ne, }}$, and the tool inclination angle is $\lambda_{\mathrm{se}}$,

$\left(\begin{array}{lll}\Delta F_{\text {snew }} \quad \Delta F_{\text {onew }} \quad \Delta F_{\text {rnew }}\end{array}\right)^{\mathrm{T}}=\mathbf{R}_{0} \mathbf{R}^{-1}\left(\begin{array}{lll}\Delta F_{\mathrm{s}} & \Delta F_{\mathrm{o}} & \Delta F_{\mathrm{r}}\end{array}\right)^{\mathrm{T}}$,

and the values of $\Delta F_{\text {snew }}, \Delta F_{\text {onew, }}$, and $\Delta F_{\text {rnew }}$ can be obtained, which are the cutting forces when the cutting edge angle is $\kappa_{\text {re }}$, the normal rake angle is $\gamma_{\text {ne, }}$, and the tool inclination angle is $\lambda_{\mathrm{se}}$.

\subsection{ECT drilling power}

In order to obtain the ECT drilling power, the three cutting forces ( $\left.\Delta F_{\text {snew }}, \Delta F_{\text {onew }}, \Delta F_{\text {rnew }}\right)$ calculated with Eq. (27) should be transformed into tangential, radical, and axial components at the position of the ECT on the main cutting edge. Assume the three components to be $\Delta F_{\mathrm{lgh}}, \Delta F_{\mathrm{mgh}}, \Delta F_{\mathrm{ng} h}$, respectively, with reference to [1], the torque $\Delta T_{g h}$ and axial force $\Delta R_{g h}$ of the ECT between node $g$ and node $h$ are, respectively,

$$
\Delta T_{g h}=\Delta F_{\operatorname{lgh}} r_{g h}, \Delta R_{g h}=\Delta F_{\mathrm{n} g h} .
$$

The drilling power $\Delta P_{g h}$ (in watts) of the $i$ th ECT, that is, the weight $w_{g h}$ of edge $\mathrm{P}_{g} \mathrm{P}_{h}$ is

$w_{g h}=\Delta P_{g h}=N\left(2 \pi \Delta F_{\operatorname{lgh}} r_{g h}+\left|\Delta F_{\mathrm{n} g h}\right| f\right)$.

Apparently, the torque $\Delta T_{g h}$ of the ECT can be deemed as the weight $w_{g h}$ of edge $\mathrm{P}_{g} \mathrm{P}_{h}$. However, what is obtained through this procedure is the main cutting edge curve with the minimal torque.

\subsection{Validation of the drilling power (force) calculation model}

In order to examine the accurateness of the drilling force and the drilling power obtained through the above procedure, the predicted results for the drilling power, the torque, and the axial force of the standard twist drill are compared to the calculation results obtained from the formulas recommended in reference [2], as shown in Table 2. The structural parameters used in the calculation are as follows: diameter: $D=$ $25 \mathrm{~mm}$; drill core thickness $2 r_{0}=3.6 \mathrm{~mm}$; , and half drill point angle: $\varphi_{0}=59^{\circ}$; chisel edge inclination angle: $\psi=55^{\circ}$; and helical angle at the outer corner $\mathrm{C}: \beta=30^{\circ}$. The workpiece material is $0.50 \%$ carbon steel. Since the tool inclination angle along the chisel edge is 0 , the cutting process is deemed as an orthogonal cutting process, and therefore $F_{\mathrm{o}}=0$. The figures in Table 2 reveal that the relative errors $\left(=\frac{\text { (predicted value }- \text { value in reference) }}{\text { value in reference }} \times 100 \%\right)$ between the predicted values of the drilling power and the torque and the calculation results from formulas in reference [2] are within $\pm 5.5 \%$, which is acceptable. This indicates that the previously proposed models of the drilling force and the drilling power are accurate.

\section{MCECMDP based on Dijkstra's algorithm}

\subsection{Program design}

The steps below describe the procedure based on Dijkstra's algorithm for determining the MCECMDP on the given rake face and calculating its drilling power (Fig. 5):

1. Input the structural parameters $\left(D, r_{0}, \varphi_{0}, \beta, \psi\right)$ of the twist drill, its rotation speed $(N)$ and feed $(f)$, the search region parameter $\left(r_{01}\right)$, and the meshing parameters $(n, m)$.

2. Do the meshing. Let $i=1,2,3 \ldots \ldots n-1$ and $j=1,2,3 \ldots \ldots$. $m$, then calculate the polar coordinates $\left(r_{i}, \theta_{i j}\right)$ and the spatial coordinates $\left(x_{i j}, y_{i j}, z_{i j}\right),\left(x_{g h m}, y_{g h m}, z_{g h m}\right)$, etc. with Eqs. (1) (5), (11).

3 Let $i=1, j=1, k=1$. 
Table 2 Comparison between the drilling power, the torque, and the axial force of the standard twist drill as predicted by the proposed models and the values calculated with the formulas recommended in reference [2]

\begin{tabular}{|c|c|c|c|c|c|c|c|}
\hline \multirow[t]{2}{*}{ Rotating speed $N / r \cdot \min ^{-1}$} & \multirow[t]{2}{*}{ Feed $f / m m \bullet r^{-1}$} & \multicolumn{2}{|c|}{ Drilling power $P / \mathrm{W}$} & \multicolumn{2}{|c|}{ Torque $T / \mathrm{N} \bullet \mathrm{m}$} & \multicolumn{2}{|c|}{ Axial force $R / \mathrm{N}$} \\
\hline & & Predicted & Reference & Predicted & Reference & Predicted & Reference \\
\hline \multirow[t]{5}{*}{300} & 0.02 & 169.978 & 165.122 & 5.409 & 5.254 & -560.205 & -608.728 \\
\hline & 0.05 & 361.191 & 364.813 & 11.488 & 11.603 & -1117.106 & -1193.738 \\
\hline & 0.08 & 531.563 & 548.031 & 16.902 & 17.423 & -1394.141 & -1686.309 \\
\hline & 0.11 & 690.701 & 722.152 & 21.956 & 22.950 & -1720.144 & -2131.03 \\
\hline & 0.14 & 842.502 & 890.060 & 26.768 & 28.275 & -2238.489 & -2544.311 \\
\hline \multirow[t]{5}{*}{450} & 0.02 & 254.980 & 244.171 & 5.409 & 5.180 & -600.799 & -591.933 \\
\hline & 0.05 & 541.859 & 539.572 & 11.489 & 11.441 & -1213.265 & -1160.803 \\
\hline & 0.08 & 797.524 & 810.643 & 16.904 & 17.181 & -1543.487 & -1639.785 \\
\hline & 0.11 & 1036.383 & 1068.273 & 21.959 & 22.633 & -1921.106 & -2072.237 \\
\hline & 0.14 & 1264.281 & 1316.723 & 26.773 & 27.887 & -2489.826 & -2474.115 \\
\hline \multirow[t]{5}{*}{600} & 0.02 & 339.985 & 322.302 & 5.409 & 5.128 & -631.793 & -580.299 \\
\hline & 0.05 & 722.552 & 712.330 & 11.490 & 11.328 & -1286.684 & -1137.989 \\
\hline & 0.08 & 1063.548 & 1070.268 & 16.906 & 17.013 & -1657.514 & -1607.556 \\
\hline & 0.11 & 1382.182 & 1410.477 & 21.962 & 22.413 & -2074.542 & -2031.508 \\
\hline & 0.14 & 1686.245 & 1738.576 & 26.778 & 27.616 & -2681.725 & -2425.488 \\
\hline Relative errors (\%) & & \multicolumn{2}{|c|}{$-5.343 \sim 5.486$} & \multicolumn{2}{|c|}{$-5.330 \sim 5.485$} & \multicolumn{2}{|c|}{$-19.281 \sim 13.066$} \\
\hline
\end{tabular}

4. Calculate $g, h, l_{i j k}, \Delta r_{i}$ with Eqs. (6) (8) and calculate $\Delta P_{g h}$ with Eqs. (9) $\sim(29)$.

5. If $l_{i j k} \leq \Delta r_{i}$, then $w_{g h}=\Delta P_{g h}$; otherwise, $w_{g h}=\infty$.

6. If $k<m$, let $k=k+1$, and go to (4); otherwise, go to (7).

7. If $j<m$, let $j=j+1$, and go to (4); otherwise, go to (8).

8 If $i<n$, let $i=i+1$, and go to (4); otherwise, go to (9).

(9) Let $\boldsymbol{A}=\left(w_{g h}\right)_{n m \times n m}$

(10) Making use of Dijkstra's algorithm, determine the various MCECMDPs (node serials):

$\Gamma_{p}=\Gamma_{S F}, \quad p=1,2,3 \ldots \ldots m$,

whose source nodes are the outer corner (with node number $S=m$ ) and whose destinations are nodes on the arc AB (numbered $F, F=n m-m+1, n m-m+2, n m-m+3 \ldots \ldots n m)$. Then, calculate their corresponding drilling power:

$P_{\min p}=P_{\min S F}, \quad p=1,2,3 \ldots \ldots m$.

Find out the main cutting edge curve

$\Gamma_{\min }=\Gamma_{p 0}, p 0 \in\{1,2,3 \ldots \ldots m\}$,

with the lowest minimal drilling power

$P_{\min }=\min \left(P_{\min p}\right)=P_{\min p 0}$.

This curve $\Gamma_{\min }$ serves as the most optimized result.

Based on the above procedure, a Matlab program is designed to determine the MCECMDP on the given rake face and its drilling power.

\subsection{Preliminary result}

In calculation, the structural parameters of the twist drill are identical to those of the standard twist drill mentioned in Section 4.6; the drilling parameters are as follows: rotation speed: $N=450 \mathrm{r} \bullet \mathrm{min}^{-1}=7.5 \mathrm{r} \bullet \mathrm{s}^{-1}$; feed: $f=0.11 \mathrm{~mm} \cdot \mathrm{r}^{-1}=$ $1.1 \times 10^{-4} \mathrm{~m} \cdot \mathrm{r}^{-1}$; discrete parameters of the rake face helicoid: $n=80, m=80$; parameters of search region: distance from straight line $\mathrm{AD}$ to $x: r_{01}=0.35 r_{0}$ and the radius of arc $\mathrm{AB}$ : $r_{0} / \sin \psi$.

\subsubsection{Minimal drilling power}

The calculated minimal drilling power of the main cutting edge $P_{\min }$ is $894.888 \mathrm{~W}$ (accurate to three decimal places, the same hereafter). The corresponding drilling torque $T$ is $19.228 \mathrm{~N} \cdot \mathrm{m}$.

Move straight line $\mathrm{AD}$ in Fig. 2 till it is infinitely close to straight line $\mathrm{BC}$, and the drilling power and the drilling force of the two main cutting edges of the standard twist drill can be obtained with the abovementioned method. The drilling power $P_{\text {standard }}$ is $962.409 \mathrm{~W}$ and the torque $T_{\text {standard }}$ is $20.753 \mathrm{~N} \cdot \mathrm{m}$.

The calculated minimal drilling power of the main cutting edge is reduced by $7.016 \%$ compared to the drilling power of the main cutting edge of the standard twist drill. 


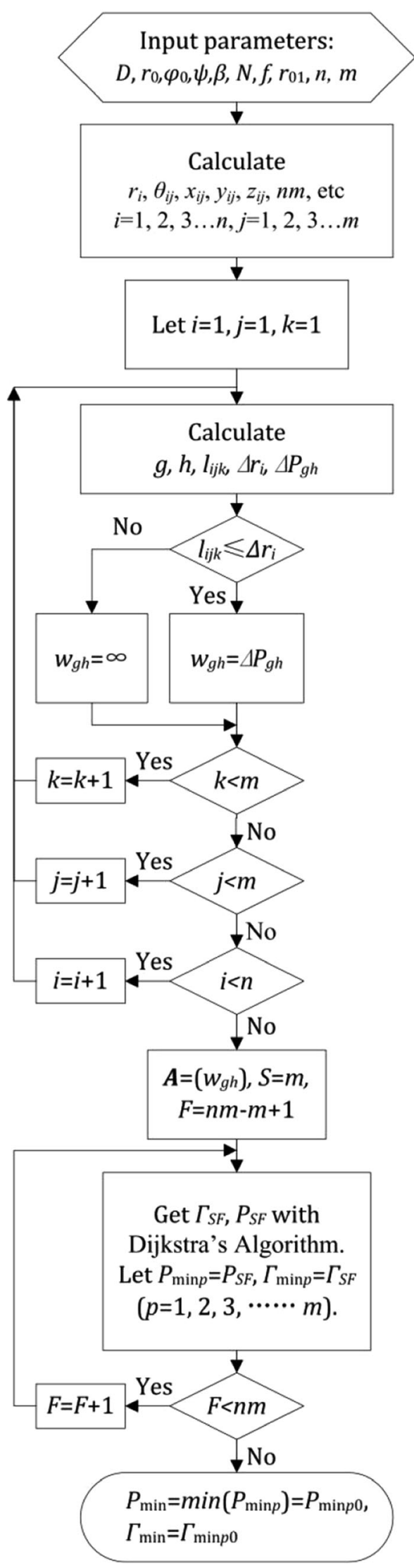

Fig. 5 Program flow chart

\subsubsection{MCECMDP}

The calculated MCECMDP and the distribution curves of the cutting angles and the drilling power are shown in Fig. 6. The following conclusions can be drawn from the figure:

1. For most points on the main cutting edge curve with the minimal drilling power, the corresponding tool inclination angle $\lambda_{\text {se }}$ is not 0 . As is known, only when $\lambda_{\mathrm{se}}=0$ does the rake angle take the maximum value [5-8]. Therefore, the MCECMDP is not the main cutting edge curve with the largest rake angles.

2. The distribution curves of the cutting angles of the MCECMDP are broken lines, among which the one for the cutting edge angle $\kappa_{\text {re }}$ is a completely zigzagged broken line. The main cutting edge curve is not smooth, either, the enlarged version of which shows that some cusps exist on the curve. Since the main cutting edge is grinded by the grinding wheel, and a single feed is unlikely to result in a cusp, the machinability of the main cutting edge resulted from the previous procedure is not satisfactory.

\subsection{Densification of the discretized grids on the rake face}

The reason for the poor machinability of the above MCEC MDP is that the discretized grids are too large.

The smoothness and the machinability of the main cutting edge can be guaranteed by eliminating the cusps on the MCECMDP and on the distribution curves of the cutting angles through grid densification.

If the grid is densified directly, for example, if the values of $n$ and $m$ are increased on the condition that the discretized region $\mathrm{ABCD}$ remains unchanged, the complexity of the calculation process will increase dramatically as the number of the nodes increases. As is known to all, when Dijkstra's algorithm is used to solve the single-source shortest path problem, its time complexity will be $O\left(p^{2}\right)$ [14] if the node number is $p$. In other words, the time complexity of the calculation process is proportional to the square of the node number. To avoid excessive time consumption, successive densification by means of gradual diminishment of the discretized region $\mathrm{ABCD}$ (Fig. 2) is adopted to solve the problem. The procedure is as follows:

1. Assign a moderately bigger value to $n$, and a moderately smaller value to $m$, for example, $\mathrm{n}=100$ and $\mathrm{m}=50$, then determine the MCECMDP. Of course, neither the main cutting edge curve or the distribution curves of the cutting angles is smooth. 
Fig. 6 Preliminary result
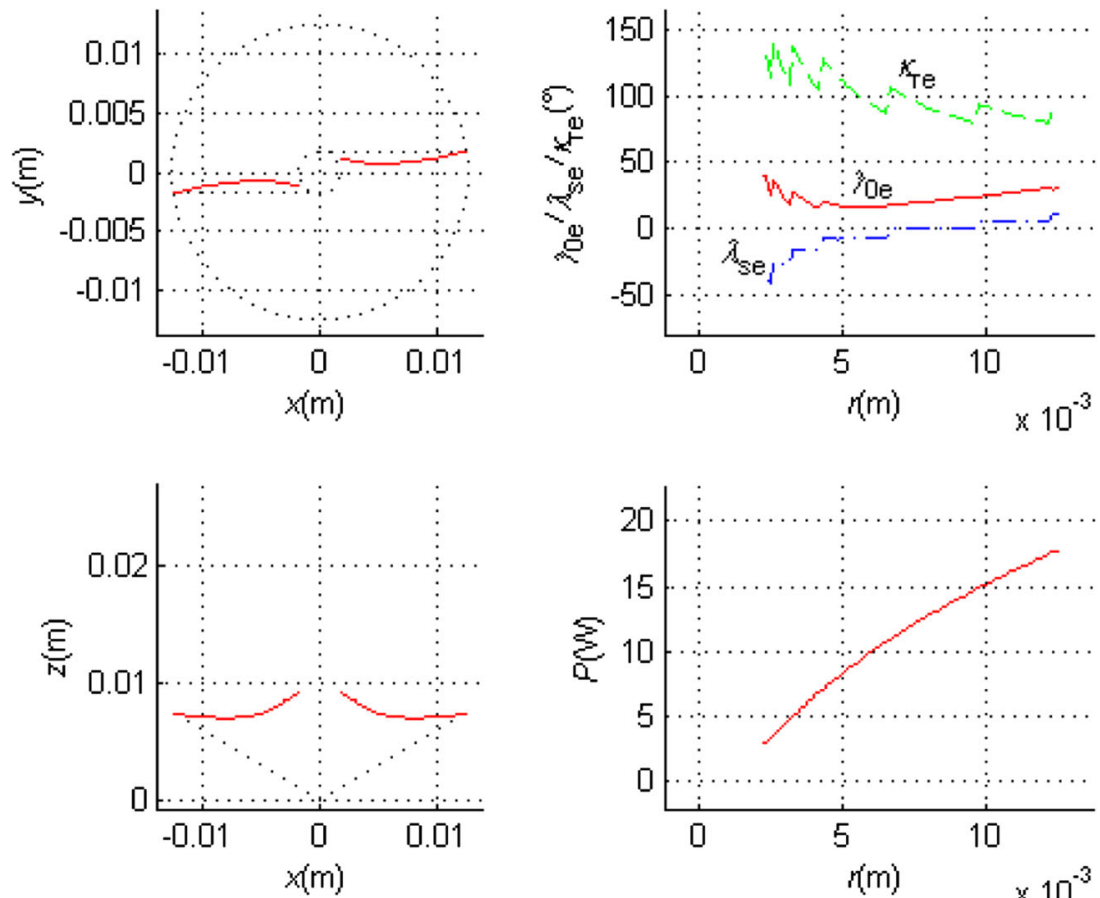

2. Assume the central angle of the arc between $\mathrm{x}$ axis and the node on the newly determined main cutting edge curve where the radius is $r_{i}$ to be $\theta_{i 0}$. Construct two curves by connecting nodes $\left(r_{i}, \theta_{i 0}\right.$-bound $)(i=1,2,3 \ldots n)$ and $\left(r_{i}, \theta_{i 0}+\right.$ bound) $(i=1,2,3 \ldots n)$, respectively, in successive order. Constrict the discretized region $\mathrm{ABCD}$ to the area formed by the two new curves and the two $\operatorname{arcs}(\mathrm{AD}, \mathrm{BC})$, with $n$ and $m$ unchanged. Determine the corresponding MCEC MDP.

3. If the main cutting edge curve and the distribution curves of the cutting angles remain broken, multiply bound by $\mathrm{k}(0<\mathrm{k}<1)$ to form a new bound. Then, return to (2). Otherwise, the calculation process comes to the end.

\subsection{Calculation results after grid densification}

Figure 7 illustrates the calculation results after the above densification procedure when $n=400, m=50$, bound $=5^{\circ}, \mathrm{k}=0.8$, and the other parameters are the same as stipulated in Section 5.2. Figure 7a shows the situation before densification, where some cusps exist apparently on the main cutting edge curve and the distribution curves of the cutting angles; Fig. 7b, c, respectively, show the results after the first and the second rounds of densification, where the curves become more smooth successively but the cusps still exist, though less apparently; Fig.7d is the result after enough rounds of densification, where all the curves have become smooth. It can be seen from the above figures that every round of densification results in moderate improvement in smoothness of the curves. Correspondingly, the calculated value of the minimal drilling power is diminished gradually until it becomes a constant as the grids are successively densified. The values of the minimal drilling power corresponding to Fig. $7 \mathrm{a}-\mathrm{d}$ are, respectively, 916.383, 912.724, 907.450, and 892.132 W. Compared to the drilling power of the straight edged standard twist drill, which is $963.941 \mathrm{~W}$, the drilling power of the curved main cutting edge is reduced by $7.450 \%$.

The following two points are worth mentioning:

1. Any point on the secondary cutting edge of the twist drill being the source point, a MCECMDP whose ending point is somewhere on the cylindrical surface of the drill core can be determined through the abovementioned method, and all these main cutting edge curves have the same spatial shape and the same drilling power. Any one of them $(l)$ can be deemed as the result of the helical motion of another ( $l$ ') round the drill spindle until it reaches the present position (of $l$ ).

2. The optimization of the main cutting edge curve can only result in a decrease of $7 \sim 8 \%$ in drilling power compared to the main cutting edge of the standard twist drill. It can be inferred that the fact that the Ni Zhifu drill and the large-rake-angle drill reduce the energy consumption in drilling by about $20 \%$ is the synthetic effect of multiple improvements in structure such as the curved main cutting edge, the chip breaker, and the shortened chisel edge, etc. 
Fig. 7 Calculation results after grid densification

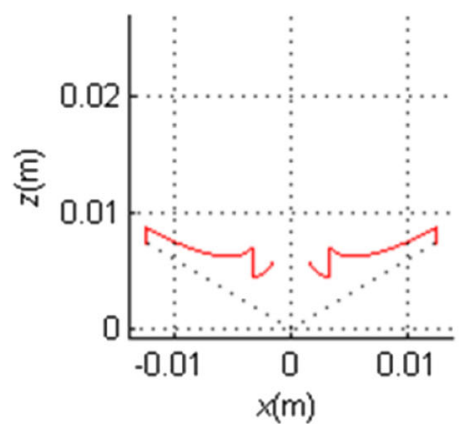

(a)
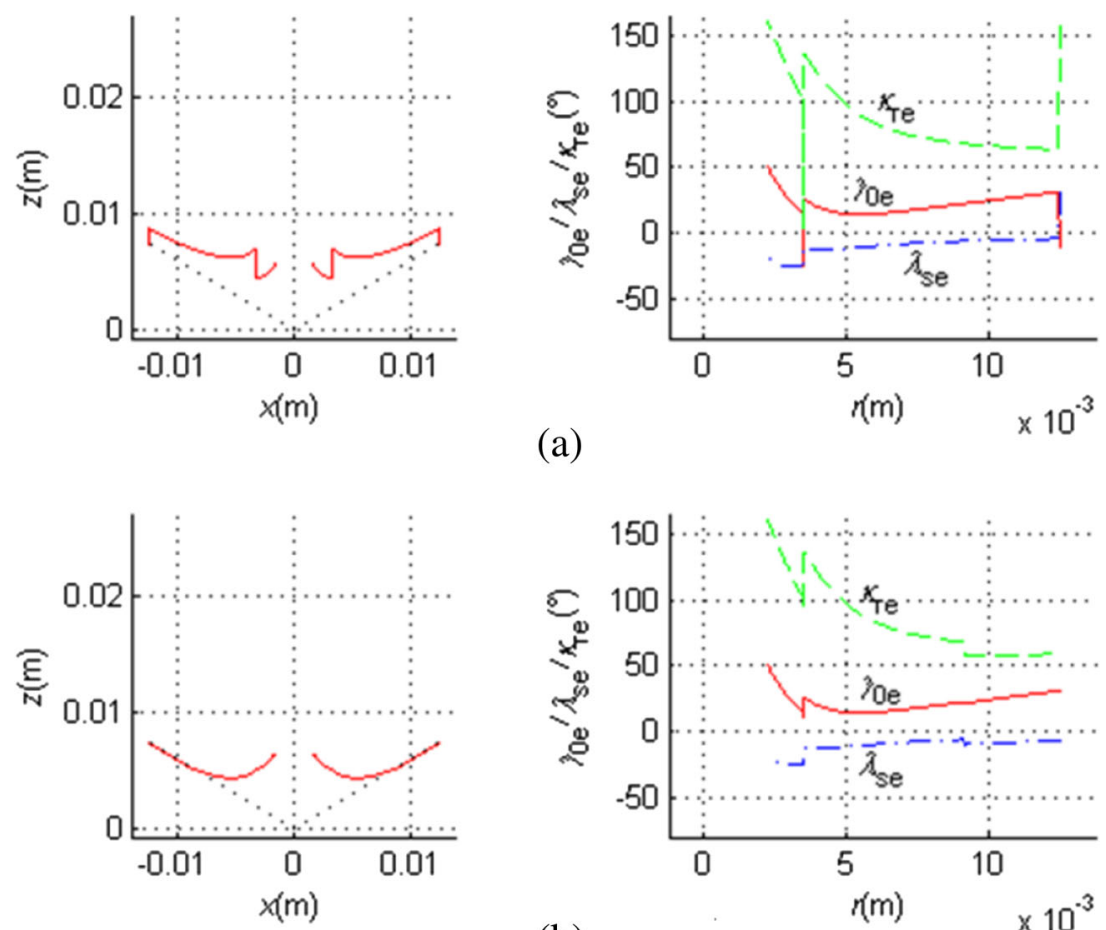

(b)
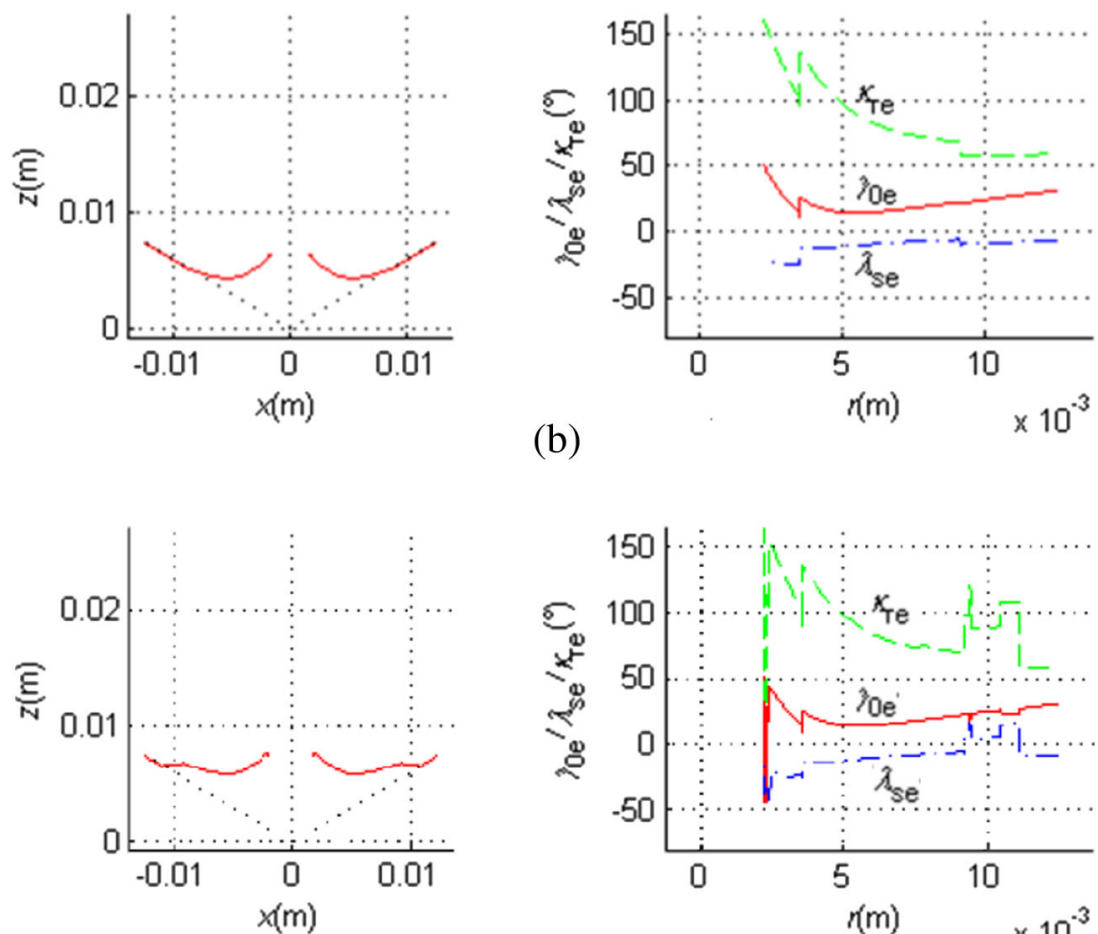

(c)
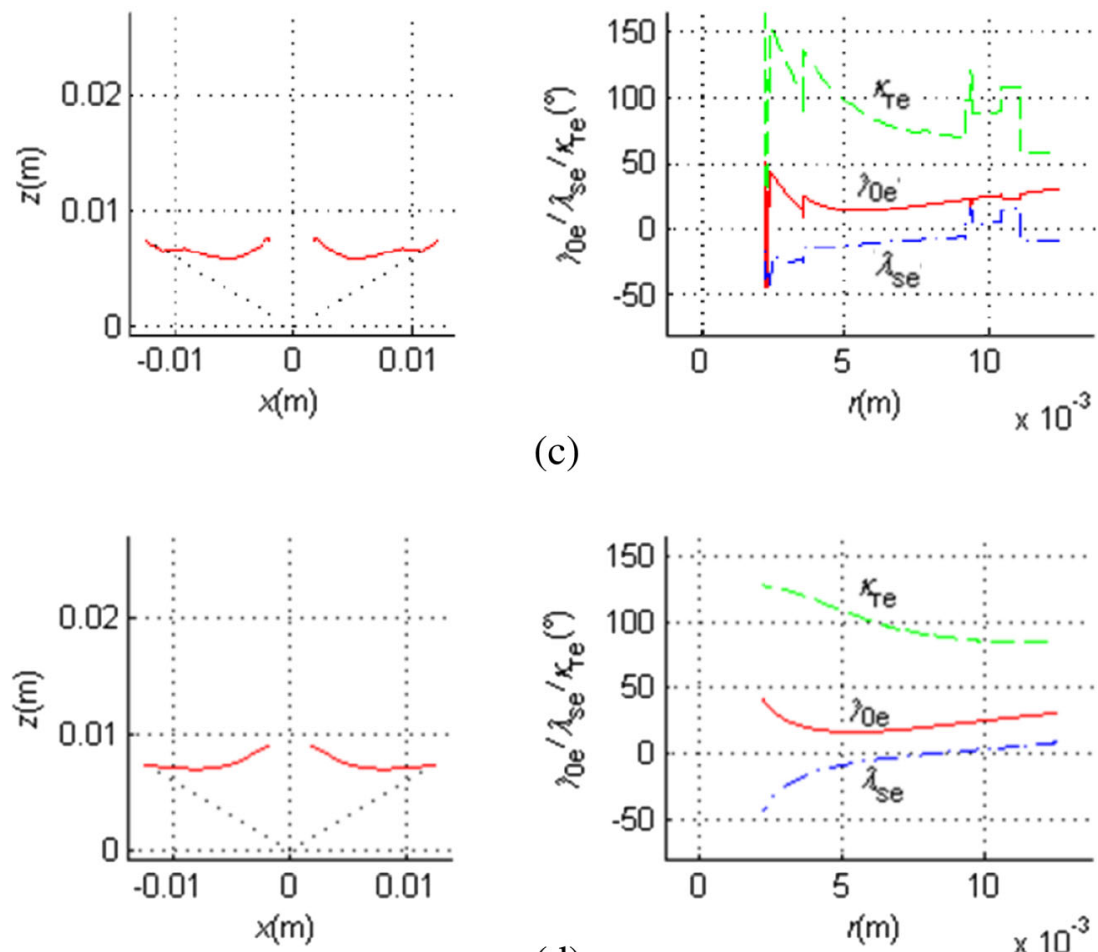

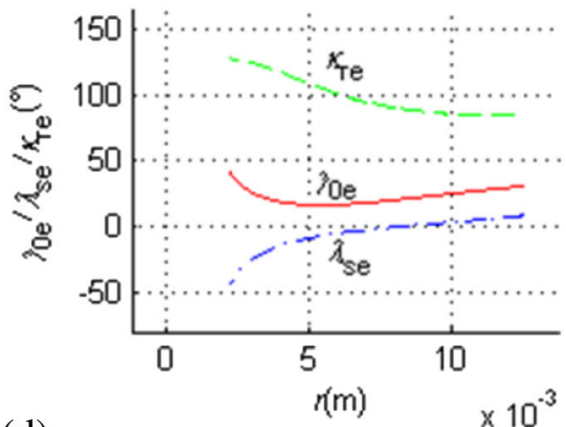

(d)

2. For a given helicoid rake face, the MCECMDP is not the one with the largest rake angles.

3. By grinding the main cutting edges of the standard twist drill as curves, the drilling power of the main cutting edge can be reduced by $7.45 \%$ at most compared to the straight edge when cutting the $0.50 \%$ carbon steel under the 
circumstance of the given cutting parameters. The fact that the MFD and the large-rake-angle drill reduce the energy consumption in drilling by about $30 \%$ is the synthetic effect of multiple improvements in structure such as the curved main cutting edge, the chip breaker, and the shortened chisel edge, etc.

4. The shortest path algorithms can be effectively used to determine the functional extremums of any curve defined in a spatial hook surface through discretization.

Acknowledgments The research in this paper was funded by the National Natural Science Foundation of China (Grant No. 51075165 and Grant No. 51121002). The related cutting experiments are conducted with the machine tools and test instruments of the State Key Lab of Digital Manufacturing Equipment \& Technology. Sincere gratitude goes to the two engineers, Mr. Wenkai Zhu and Mr. Qingshan Li, who operated the machine tools for the cutting experiments and the three graduate students, Ge Yi, Huasheng Bi, and Ruofeng Huang, who took part in the cutting experiments, recorded and processed the experimental data.

Open Access This article is distributed under the terms of the Creative Commons Attribution 4.0 International License (http:// creativecommons.org/licenses/by/4.0/), which permits unrestricted use, distribution, and reproduction in any medium, provided you give appropriate credit to the original author(s) and the source, provide a link to the Creative Commons license, and indicate if changes were made.

\section{References}

1. Wang JL (1994) Development of new drilling force models for improving drill point geometries. The University of Michigan, Michigan

2. Ni ZF, Chen GB (1999) Multiface drill—Ni Zhifu drill. Shanghai Science and Technique Publishing House, Shanghai

3. Paul A, Kapoor SG, DeVor RE (2005) Chisel edge and cutting lip shape optimization for improved twist drill point design. Int J Mach Tools Manuf 45:421-431

4. Sambhav K, Dhande SG, Tandon P (2010) CAD based mechanistic modeling of forces for generic drill point geometry. Comput Aided Des Appl 7:809-819
5. Shi HM (1982) A new method for analyzing and calculating angles on cutting tools. Int J Mach Tool Des Res 22:177-196

6. Shi HM (1986) Graphic determination of geometric angles on metal-cutting tools. Int J Mach Tool Des Res 26:99-112

7. Xiong LS, Shi HM, Zhang HS (1982) A twist drill with smaller cutting force and lower energy consumption. J Huazhong Univ Sci Technol 19(II):89-94

8. Shi HM, Zhang HS, Xiong LS (1994) A study on curved edge drills. J Eng Ind Trans ASME 116:267-273

9. Xiong LS, Shi HM, Chen YJ (2006) Applying plannar displaying method to the design of flanks of curved edge drills. In: Jia ZY, Wang XY, Guo DM, Wang J (eds) Current development in abrasive technology-proceedings of the 9th International Symposium on Advances in Abrasive Technology. Frontiers of Design and Manufacturing Sydney Australia, Australia, pp 493-499

10. Xiong LS, Shi HM, Chen YJ (2007) Regrinding the cylindrical flank of curved edge drills. Chin Mech Eng 18(1165-1167):1192

11. Xiong LS (2006) Research on optimization of cutting edge shapes of twist drills and the sharping methods. Huazhong University of Science and Technology, Wuhan

12. Xiong LS, Fang N, Shi HM (2009) A new methodology for designing a curve-edged twist drill with an arbitrarily given distribution of the cutting angles along the tool cutting edge. Int $\mathrm{J}$ Mach Tools Manufact 49:667-677

13. Wald RM (2010) General relativity. University of Chicago press

14. Dijkstra EW (1959) A note on two problems in connexion with graphs. Numer Math 1:269-271

15. Bertsekas DP (1998) Network optimization: continuous and discrete models. Athena Scientific, Belmont

16. Xin SQ, Wang GJ (2009) Improving Chen and Han's algorithm on the discrete geodesic problem. ACM Trans Graph (TOG) 28:104

17. Song Q, Wang XF (2012) Survey of speedup techniques for shortest path algorithms. J Univ Electron Sci Technol Chin 41(2):176-184

18. Geometry of the active parts of cutting tools - general terms, reference systems, tool and working angles, ISO/DIS3002.

19. Watson AR, Williams RA (1977) Specification of the cutting geometry of single point tools and twist drills using the ISO system. Int J Mach Tool Des Res 17:103-116

20. Startz R (2007) EViews illustrated for version 6. Quantitative Micro Software, Irvine

21. Liu JH, Zhang GP, Shi QF, Zou TX, Zhou S (2009) A new simulation method of cutting force model in turning. J Xi' an Univ Technol 25:351-355 